\title{
Acute fibrinous organizing pneumonia: a rare lung pathology
}

\author{
Subodh Kumar ${ }^{1,2}$, Mayank Mishra ${ }^{2}$, Kashyap Goyal ${ }^{2}$, Ritisha Bhatt ${ }^{2}$, Prashant Joshi ${ }^{3}$, Saloni Bansal ${ }^{3}$ \\ ${ }^{1}$ Department of Trauma and Emergency, All India Institute of Medical Sciences, Rishikesh; ${ }^{2}$ Department of Pulmonary \\ Medicine, All India Institute of Medical Sciences, Rishikesh; ${ }^{3}$ Department of Pathology, All India Institute of Medical \\ Sciences, Rishikesh, India
}

\begin{abstract}
Acute fibrinous organizing pneumonia (AFOP) is a rare, noninfective lung pathology histologically characterized by patchy distribution of intra-alveolar fibrin "balls" and organizing pneumonia. It needs to be differentiated from other forms of acute pneumonia like community acquired pneumonia (CAP) because the treatment modalities are contrastingly different. Interesting case of a 45-year-old male initially suspected as a case of acute bacterial pneumonia but finally diagnosed as case of AFOP, is being reported. Initial empirical antimicrobial therapy was ineffective, and the main treatment to which the patient responded was corticosteroids.
\end{abstract}

Correspondence: Mayank Mishra, Department of Pulmonary Medicine, All India Institute of Medical Sciences, Rishikesh-249203, Uttarakhand, India.

Tel.+91.9478116891. E-mail: drkashyapgoyal31@gmail.com

Contributions: MM, study concept and design, manuscript editing; $\mathrm{KG}$, manuscript drafting; RB, data acquisition; PJ, manuscript editing; SB, literature search; SK, manuscript review. All Authors read and approved the final version of the manuscript.

Conflict of interest: The Authors declare no conflict of interest.

Funding: This research did not receive any specific grant from funding agencies in the public, commercial, or not-for-profit sectors.

Patient consent: Written, informed consent was obtained from the patient.

Key words: Acute fibrinous organizing pneumonia; fibrin balls; organizing pneumonia.

Received for publication: 13 December 2018.

Accepted for publication: 27 May 2019.

(C) Copyright S. Kumar et al., 2019

Licensee PAGEPress, Italy

Monaldi Archives for Chest Disease 2019; 89:1016

doi: 10.4081/monaldi.2019.1016

This article is distributed under the terms of the Creative Commons Attribution Noncommercial License (by-nc 4.0) which permits any noncommercial use, distribution, and reproduction in any medium, provided the original author(s) and source are credited.

\section{Introduction}

Acute fibrinous organizing pneumonia (AFOP) is a newly evolving rare non-infectious lung pathology, first described by Beasley et al. in 2002 [1]. It is typically characterized histologically by the presence of intra-alveolar fibrin "balls" and organizing pneumonia in a patchy distribution. AFOP may be idiopathic or linked to a variety of causes like autoimmune disease, connective tissue disorders, drug and occupational exposures and infectious agents. It clinically presents as an unusual pattern of acute lung injury posing a great diagnostic challenge, and needs to be differentiated from CAP and other well-recognized histologic patterns such as diffuse alveolar damage (DAD) and cryptogenic organizing pneumonia (COP). Two forms of the disease are described: an acute form with a fulminant course and rapid progression to respiratory failure, and a sub-acute form with a better outcome [2]. In this article, we present the case of a 45-year-old male diagnosed with the sub-acute form of the disease, who recovered clinically after administration of steroids.

\section{Case Report}

A 45-year-old male, chronic smoker and a farmer by occupation, presented to the pulmonary outpatient department with the chief complaints of productive cough for last one month, intermittent fever, breathlessness and right sided chest pain for the last 8 days, and hemoptysis for the last 5 days. There was no history of any comorbid illness in the past, nor any history of exposure to chemicals, fumes or allergens at his home or workplace. General physical examination revealed a conscious and oriented patient with heart rate of 98 beats/min, respiratory rate $26 /$ min, oxygen saturation $84 \%$ on ambient air, temperature $101.6^{\circ} \mathrm{F}$, and pitting pedal edema. Other parameters were unremarkable. Auscultation revealed crepitations over the right infra-scapular and infra-axillary areas. Investigations showed an elevated total leukocyte count, and negative results of sputum for acid fast bacilli (AFB), Gram staining and malignant cells. Blood and urine cultures were sterile, and all other hematological, biochemical and immunological tests were negative. Chest X-ray was suggestive of non-homogenous opacities in the lower lobes of both lungs, more on right side (Figure 1a).

A provisional clinico-radiological diagnosis of right sided consolidation possibly due to CAP was proposed. Patient was started with non-invasive ventilation (NIV), supplemental oxygen and combination antibiotic therapy (piperacillin-tazobactam with linezolid). However, the patient did not respond. A 
fiberoptic bronchoscopy was planned which exhibited no endobronchial abnormality and broncho-alveolar lavage was negative for AFB, with negative culture for bacteria and fungi.

Subsequently, computed tomography (CT) scan-guided transthoracic lung biopsy was performed from the right lower lobe. CT-imaging revealed predominantly right-sided lower lobe dense consolidation with air bronchogram and few cystic changes (Figure $1 \mathrm{~b}, \mathrm{c}$ ). Histopathological examination showed diffuse intra-alveolar fibrinous deposition in the lung parenchyma with no significant inflammatory infiltrate which was suggestive of AFOP (Figure 2a-f). Since this was a fairly new clinical entity, thorough literature search was performed before initiating treatment with systemic steroids, bronchodilators and inhaled oxygen. Dramatic clinico-radiological response was noted over the next five days in the form of gradual reduction and eventual cessation of supplemental oxygen, significant improvement in frequency of cough, amount of phlegm, and breathlessness, as well as in opacities on imaging (Figure $1 \mathrm{~d}, \mathrm{e}$ ). Patient was discharged under satisfactory condition and was kept on follow up. Steroids were tapered gradually over a period of three months.

\section{Discussion}

AFOP is a relatively new clinical entity with not more than 120 cases reported till 2016 [1]. It manifests histologically as a rare pattern of acute lung injury characterized by intra-alveolar accumulation of fibrin associated with organizing pneumonia consisting of intraluminal loose connective tissue within the alveolar bronchioles and ducts [2]. This typical histological picture distinguishes it from other histologically distinct entities like COP, DAD or eosinophilic pneumonia $[3,4]$. AFOP may be idiopathic or linked to various etiological factors as listed in Table 1.

In our case, there was no evidence of any associating factor relating to AFOP. The disease has been described across all age groups and can present with markedly variable severity and mortality. In a study by Gomes et al. on 13 histologically confirmed cases of AFOP, the most common clinical presentation was fever (69.2\%), followed by cough $(46.2 \%)$, chest pain $(30.8 \%)$, constitutional symptoms (23.1\%) and dyspnea (15.4\%) [5]. Our patient presented with cough, breathlessness, fever, chest pain and hemoptysis, and skiagram chest showed bilateral lower lobe infiltrates, more on right side. Since this clinico-radiological presentation is usually encountered in CAP, it was considered as a diagnostic possibility initially. However, lung biopsy revealed the unusual histological pattern of AFOP.

Based on the clinical course of our patient, we conclude that he had the sub-acute form of AFOP. Sub-acute AFOP is often mistaken with COP. Therefore, histological diagnosis is essential for distinguishing this condition from other mimics. Numerous treatment modalities have been used for AFOP. The most commonly used and effective modality is corticosteroids. Other
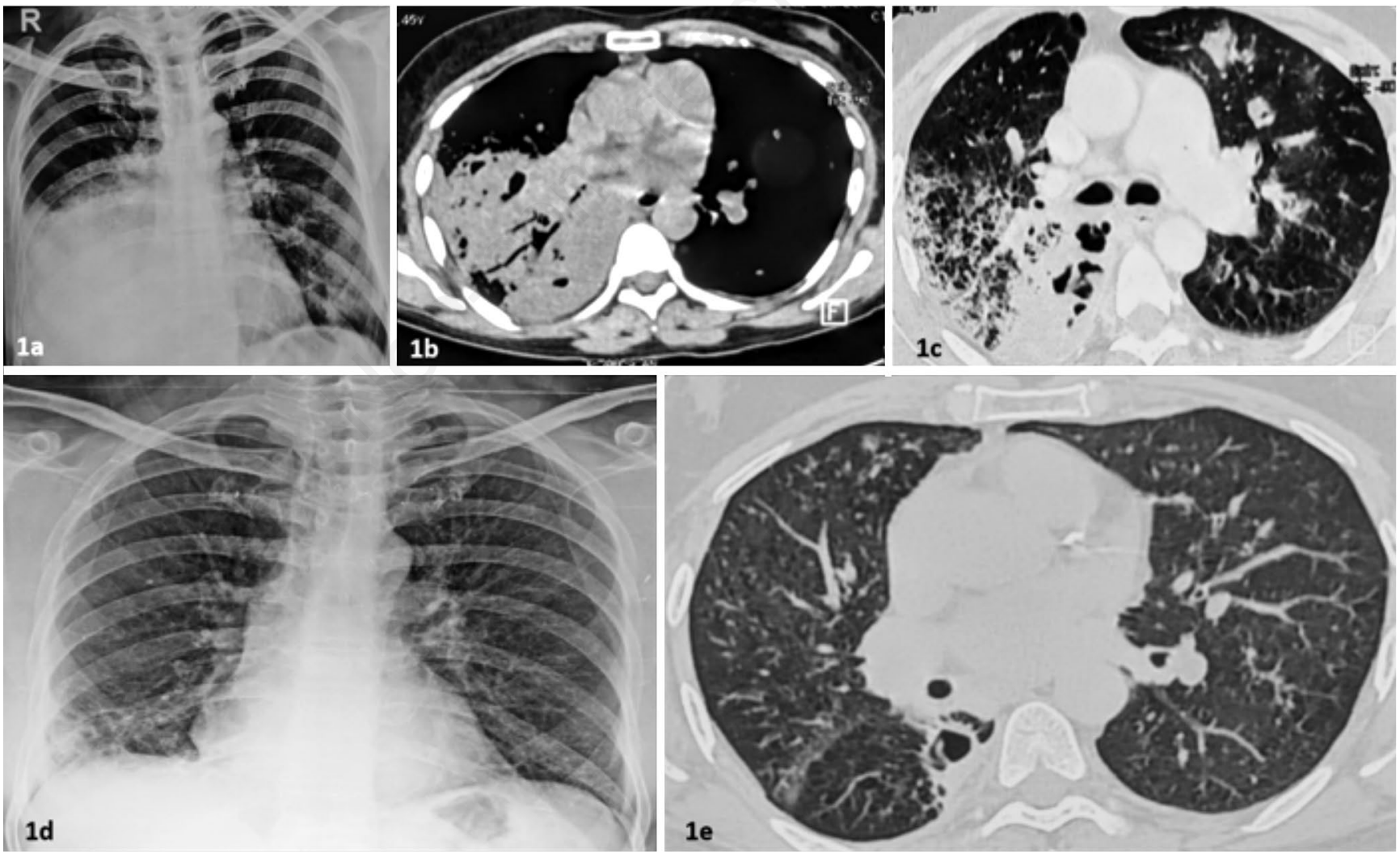

$1 d$

Figure 1. a) Chest X-ray postero-anterior view showing non-homogenous opacities in both lower lung zones, more on right side. Axial CT section, mediastinal window (b) and lung window (c), showing dense consolidation with air bronchogram and few cystic changes in right lower lobe. Chest X-ray postero-anterior view (d) and axial CT section lung window (e) showing near-complete resolution of right lower lobe opacities after treatment. 
Table 1. Possible etiological factors in acute fibrinous organizing pneumonia.

\begin{tabular}{ll} 
Autoimmune disease & Polymyositis, dermatomyositis, rheumatoid arthritis, ankylosing spondylitis \\
Drugs & Statins, bleomycin, abacavir, busulfan \\
\hline Hematological disorders & Lymphomas, acute lymphoblastic leukemia, acute myeloblastic leukemia, myelodysplastic syndrome \\
Occupational exposure & Coal mines, hair spray, zoological work \\
\hline Immune status & Human immunodeficiency virus (HIV) infection, corticosteroid therapy, uncontrolled diabetes mellitus, alcoholism \\
\hline
\end{tabular}

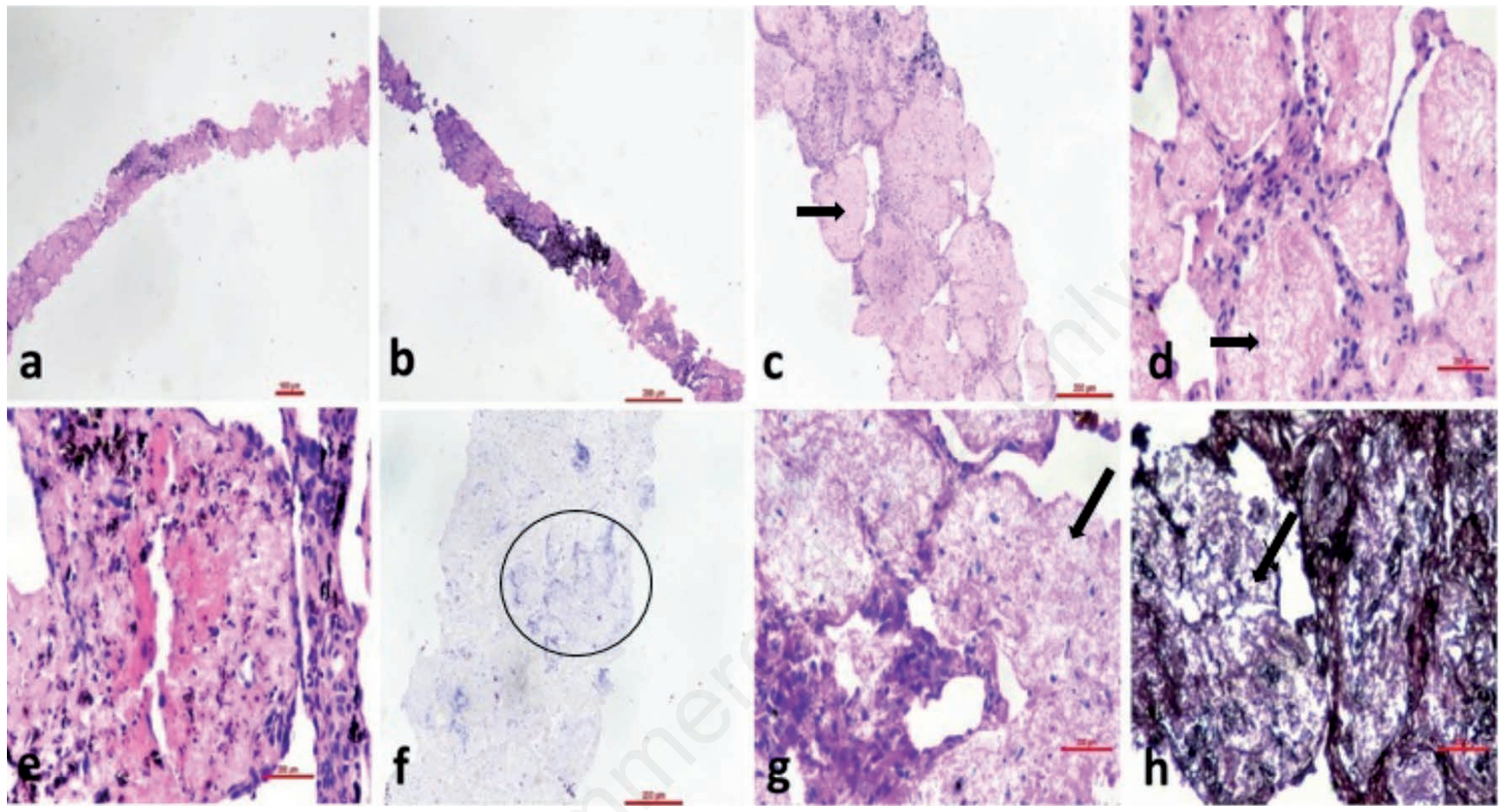

Figure 2. Sections examined from both the lung biopsy cores (a,b; Hematoxylin and Eosin (H\&E) stain, magnification 20x) show diffuse intra alveolar fibrin balls without significant inflammation (c,d; H\&E stain, magnification 100x and 400x, respectively). Arrows in c, d, $\mathrm{g}$, h show the fibrin "balls". The fibrin has stained blue with Phosphotungstic Acid Hematoxylin stain (encircled portion in f; magnification 100x) while being negative on Periodic Acid Schiff (g; magnification 400x) and Silver Methenamine (h; magnification 400x) stains. Occasional focus of residual haemorrhage is also present (e; H\&E, magnification 400x).

agents that are in use include cyclophosphamide and azathioprine, with little role of antibiotics [6]. Mechanical ventilation is often required to relieve the severe respiratory distress associated with AFOP, especially in the acute form of the disease. Our patient showed considerable clinical improvement with steroid therapy, supplemental inhaled oxygen and NIV support.

\section{Conclusions}

This case report highlights the point that AFOP should be taken into consideration as a differential diagnosis of severe acute pneumonias in patients with no significant co-morbidities. Diagnosis is mostly biopsy proven, and treatment using corticosteroids is quite promising.

\section{References}

1. Saxena P, Kumar K, Mittal S, et al. Acute fibrinous and organizing pneumonia: A rare form of nonbacterial pneumonia. Indian J Crit Care Med 2016;20:245-7.

2. Beasley MB, Franks TJ, Galvin JR, et al. Acute fibrinous and organizing pneumonia: A histological pattern of lung injury and possible variant of diffuse alveolar damage. Arch Pathol Lab Med 2002;126:1064-70.

3. Cordeiro CR. Airway involvement in interstitial lung disease. Curr Opin Pulm Med 2006;12:337-41.

4. Beasley MB. The pathologist's approach to acute lung injury. Arch Pathol Lab Med 2010;134:719-27.

5. Gomes R, Padrao E, Dabo H, et al. Acute fibrinous and organizing pneumonia. A report of 13 cases in a tertiary university hospital. Medicine (Baltimore) 2016;95:e4073.

6. Kuza C, Matheos T, Kathman D, Heard SO. Life after acute fibrinous and organizing pneumonia: A case report of a patient 30 months after diagnosis and review of the literature. J Crit Care 2016;31:255-61. 
